\title{
THE CEREBELLAR NETWORK: ATTEMPT AT A FORMALIZATION OF ITS STRUCTURE
}

\author{
V. Braitenberg \\ Max-Plank-Institut for Biological Cybernetics, Spemannstr. 38, 7400 Tübingen, Germany
}

Modellers of brain function often make little use of information from neuroanatomy. Sometimes they refer to neural structures in the brain secondarily, projecting into the neuroanatomy schemes of computation which they had first developed from quite different considerations. I doing so, they pay litue regard to the question whether the neuroanatomy by which they illustrate their abstract scheme is necessarily such as it is or could well be otherwise. We have seen the idea of the "perceptron" used as an explanation of the cerebral cortex, or of the hippocampus, or of the cerebellar cortex. One and the same idea could hardly provide a cogent explanation for the structure of such widely different networks.

My tendency is to take information from neuroanatomy more seriously. The structure of different regions of the grey substance provides precise constraints on the neural interactions which take place there even if the physiological nature of these interactions is not known in full detail. I have shown in previous papers (Braitenberg \& Atwood 1958. Braitenberg 1961, 1967; Braitenberg \& Onesto 1962. Braitenberg 1983) that in the cerebellar cortex inferences from structure to function come particularly easy and I shall carry this one step farther here.

\section{Symmetries}

The cerebellar cortex has translational symmetry in the latero-lateral as well as in the front-to-back direction. If there is a fibre running from a point A to a point $\mathrm{B}$, a certain distance to its right (or left) there is a similar fibre running from point B to another point $C$, separated from B by the same distance along the same direction (barring, of course, the situation at the margin of the contex). Similarly, the fibres running forward or backward from any point of the cerebellar surface are the same everywhere.

There is also mirror symmetry. For every fibre running to the right there is a similar fibre to the left. For every fibre running forward there is a similar fibre running in the opposite direction (again, with due respect to the margin of the cerebellar sheet).

There is no rotational symmetry, however, except for rotations which are multiples of $180^{\circ}$. The great majority of fibres in the cerebellar cortex are parallel or antiparallel to either the left-to-right of the front-to-back direction. We may formalize these anatomical relations and thus obtain general statements about the interaction of signals in the cerebellar cortex (the arrows signify synaptic influences, $x$ is the left-to-right axis, $y$ is the back-to-front axis).

$$
\begin{array}{ll}
\text { Reflexivity (mirror symmetry): } & x_{i} \rightarrow x_{j}=x_{j} \rightarrow x_{i} \\
& y_{i} \rightarrow y_{j}=y_{j} \rightarrow y_{i} \\
& \\
& x_{i} \rightarrow x_{j}=x_{i}+\Delta x \rightarrow x_{j}+\Delta x \\
& y_{i} \rightarrow y_{j}=y_{i}+\Delta y \rightarrow y_{i}+\Delta y
\end{array}
$$

(with no exception at the midline!)

It is important to note that all of these synaptic influences in the cerebellar cortex are mediated by relatively short fibres: a few millimeters in the $x$ direction, even less in the $y$ direction. Contrary to the cerebral cortex, interactions in the cerebellum are essentially local.

\section{Unidimensional layout}

The fibres oriented in the two orthogonal directions in the cerebellar cortical plane are very different in their form and physiological characteristics. Eccles et al. (1967) gave proof of the excitatory nature of "parallel fibres" which form the bulk of the population of fibres oriented parallel to the $x$-axis, and the vast majority of all fibres in the cerebellum. Eccles also showed that the axons of stellate and basket cells which form the population of fibres parallel to the y-axis are inhibitory. Hence the interaction of the elements are radically different in the two axes of the cerebellar cortical plane: summation and reciprocal enhancement are to be expected in the latero-lateral direction $x$ while along the antero-posterior direction $y$ we expect competition between elements and suppression of the weaker signal. In a functional context it 
would be wrong, therefore, to talk of the cerebcllar cortex as of a two-dimensional computer. Rather, we may describe it as essentially onc-dimensional, as an array of linear elements - bundles of parallel fibres mediating the excitatory interaction - separated from each other by inhibitory influences. The more activity spreads laterally through the bundles or beams of parallel fibres, the more it will stand out on the background of the silenced neighbouring bundles.

\section{Metric invariants and timing}

The variety of animals makes it possible to define the structural invariants of an organ that is shared by many species, the structural invariants of the vertebrate leg, for instance, or of the liver. We may describe the essence of an organ as the set of properties which are common to all instances of that organ in different species, and to all parts of it. If we do this for identified portions of the vertebrate brain, for the striatum, for example, or for the cerebral cortex, we discover structural invariants which refer to the layout of neurons and fibres, to the patterns formed by their intertwined processes, to the shape of individual dendritic and axonal ramification. These are specified mostly in terms of a "topological" description, where neighbourhood matters, but distances may be grossly distored between one part of the structure and another. For example, the layers of the cerebral cortex form a succession which is quite constant and characteristic for that part of the brain, but their relative thickness varies a great deal depending on their location or the summit of a convolution or in the depth of a furrow.

Metric, rather than topological invariants, are the exception, and when they occur they point to an unusual importance of distances in the function of a piece of nerve tissue. In the cerebellum, the molecular layer preserves a remarkably constant thickness in spite of the rich folding of the cerebellar cortex. Also, with all the folding, the "parallel fibres" remain remarkably straight and the planar dendritic trees of Purkinje cells, which are arranged at right angles to the parallel fibres, stay parallel to each other. These metric invariants led to a model of the cerebellar cortex in which measurement of time intervals between successive signals (with a resolution of about $0.1 \mathrm{~ms}$ ) played the central role (Braitenberg \& Alwood 1958).

\section{Transformation of input by the parallel fibres}

The vast majority of cerebellar fibres (several orders of magnitude more than the other elements) are represented by the "parallel fibres". These relay the (mossy fibre) input from any point of the cercbellar cortex a few millimeters to either side along the $x$ direction, passing it on to all the other neurons which they meet on their way (mainly Purkinje cells and various kinds of stellate cells). The "molecular layer" of the cerebellar cortex is essentially a thick felt of oriented fibres whose main function scems to be to smear out the input in space and in time in an anisotropic fashion.

The activity $A(x, y, t)$ generated in the cerebellar cortex by the input $I(x, y, t)$ via the parallel fibres $P$ which conduct at a speed $v_{0}$ can thus be expressed by a convolution of I with $P$

$$
A=I * P
$$

or explicitly

$$
A(x, y, t)=\iint 1(x \pm \xi, y, t-\tau) P(\xi, \tau) d \xi d \tau
$$

00

Since the function $\mathrm{P}(\xi, \tau)$ may be simply taken as

$$
P(\xi, \tau)=\left\{\begin{array}{l}
1 \text { for } \xi=\tau v_{0} \\
0 \text { otherwise }
\end{array}\right.
$$

this reduces to

$$
A(x, y, l)=\int_{0}^{\hat{\tau}} I\left(x \pm v_{0} \tau, y, l-\tau\right) d \tau
$$


We may represent also the inhibition coming from neighbouring rows (or beams, or grooves) at a distance $\Delta y$ as simply opposing the activity and get

$$
A(x, y, t)=\int_{0}^{\hat{\tau}} I\left(x \pm v_{0} \tau, y, t-\tau\right) d \tau-A(x, y \pm \Delta y, t)
$$

\section{Tidal waves}

Considering only one strip of cerebellar cortex oriented in the direction of the $x$ axis, a "beam of parallel fibres" in Eccles' terminology, the transformation of the input into the output can be simply described as lateral propagation through parallel fibres from every point a few millimeters to the right and to the left at a velocity $v_{0}$. Estimates for the length of parallel fibres vary between 2 and $5 \mathrm{~mm}$ on each side, and conduction velocities measured were about $0.5 \mathrm{~m} / \mathrm{s}$ in mammals and $0.3 \mathrm{~m} / \mathrm{s}$ in frogs. We assume a length $\xi_{\max }=4 \mathrm{~mm}$ and a velocity $v_{0}=0.5 \mathrm{~m} / \mathrm{s}$, which gives as a maximum delay $\tau_{\max }=$ $\xi_{\max } / v_{0}=8 \mathrm{~ms}$ generated by an individual parallel fibre between the input and the output-(Purkinje) cell.

Such delays of a few milliseconds may be involved in some neuronal information handling schemes especially in sensory contexts, but they are of little use in motor physiology, where the cerebellum is undoubledly involved. The episodes of motor behaviour whose coordination is severely impaired when the cerebellum is defective typically have durations of a few hundred milleseconds. The idea of the cerebellum generating the delays between the activation of an agonist muscle (e.g. biceps) and of an antagonist (e.g. triceps) starting and stopping a movement (e.g. flexion of the forearm) has its merits but cannot be related to cerebellar parallel fibres in the simple form which was favored in the past.

However, even if individual parallel fibres are 100 short to generate significant delays, the tissue which is formed by their staggered arrangement has a width greatly excceding their length, especially in larger animals. From one side of the cerebellum to the other, a beam of parallel fibres in the human cerebellum may have a length of over $10 \mathrm{~cm}$. With the known velocity $v_{0}$ of conduction in parallel fibres, a signal travelling from one side of the cerebellar cortex to the other would take a few hundred milliseconds, a time well compatible with the organisation of movement. Is there any circuitry in the cerebellum that could relay signals from one parallel fibre to the next along a "beam", thus generating the long delays which we need?

There is not, but if we shift our attention from the idea of "generation of delay" to that of "discovery of temporal sequences" we can still make good use of the width of cerebellum in terms of the organisation of movement.

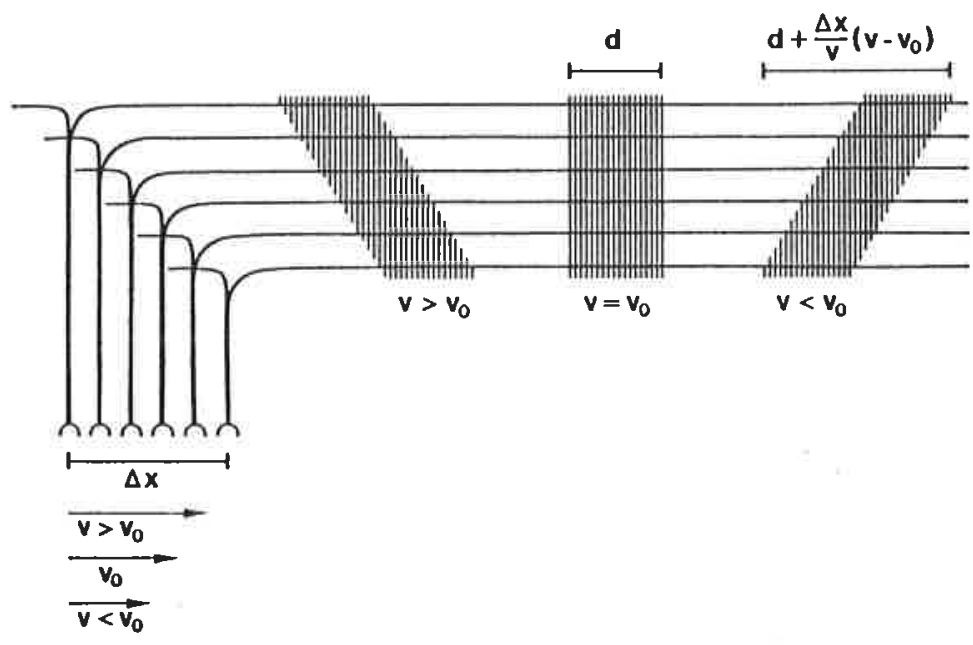

Fig. 1 
The idea is to consider the molecular layer of the cerebellar cortex as a whole as a tissue conducting signals at the velocity $v_{0}$ (of conduction in parallel fibres, of which it is composed) in the latero-lateral direction. If such a conducting medium is excited by a stimulus which moves (in the appropriate direction) at the velocity of the intrinsic velocity $v_{0}$, a sort of tidal wave will be elicited in the tissue and the response will be maximal, for the moving stimulus will add continually to the excitation which already propagates through the tissue. For velocities of the input layer larger or smaller than $v_{0}$ there will be less of a tidal wave. The dependence can be described quantitatively (fig. 1)

Let the input be a local stimulus moving along the bcam of parallel fibres at the velocity $v$ over a length $\Delta \mathbf{x}$. The amount of cxcitation which it sets up at each input point is constant and independent of $v$, hence the total excitation produced is $E \sim \Delta x$. $A$ is the aclivity elicited in the cerebellum, which we define as the density of excitation per unit length of the beam. This depends of course on the spread d of the individual wave of excitation moving along a parallel fibre, and on the dispersion of the excitation in the ensemble of parallel fibres which occurs when the velocity of the stimulus $v$ is different from the intrinsic velocity $v_{0}$. The dispersion is proportionate to the time it took the stimulus to travel a length $\Delta x$, and to the (positive) difference between intrinsic and extrinsisc velocity. The expression

$$
A=\frac{E}{\frac{\Delta x}{v}\left|v-v_{0}\right|+d}
$$

is plotted in fig. $2 \mathrm{a}$ as a function of $\mathrm{v}$ for $\Delta \mathrm{x}=\mathrm{d}$. Fig. $2 \mathrm{~b}$ shows the same for $\Delta \mathrm{x}=10 \mathrm{~d}$. Evidently, the cerebellar cortex is a sensitive detcctor for signals moving at a certain velocity over the array of its input fibres. But is it used as such?
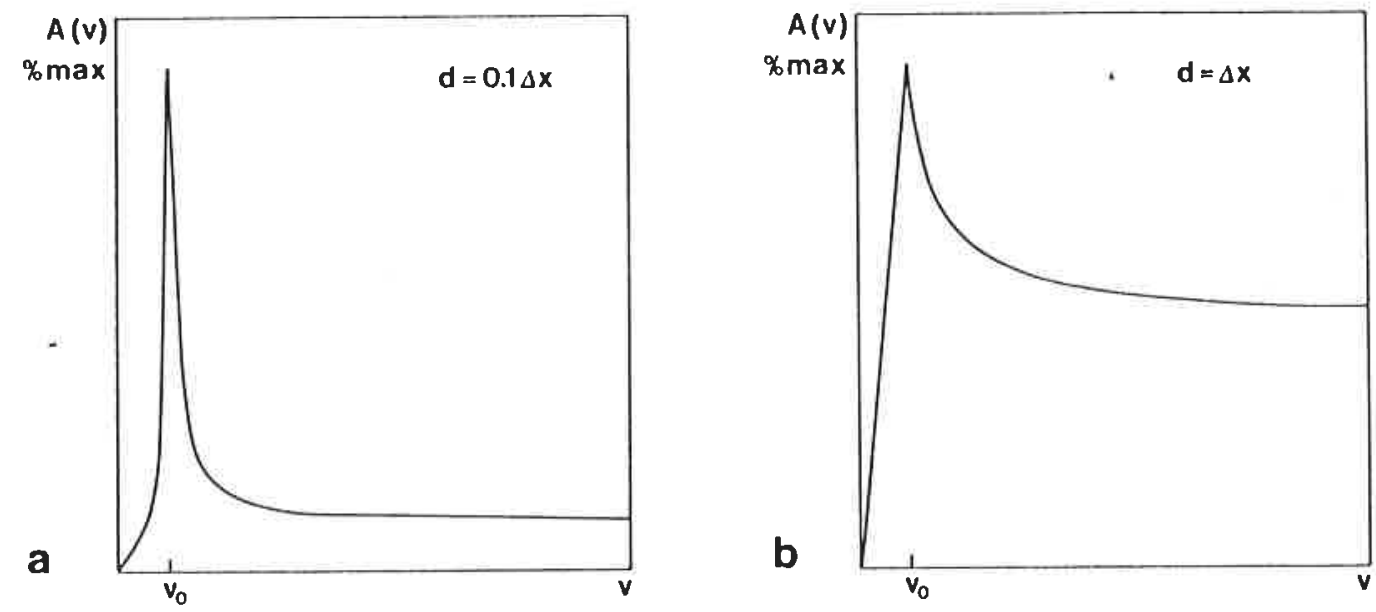

Fig. 2

The question remains open, because not enough is known about the insertion of the cerebellum into the rest of the motor (or, generally, nervous) system. However, we can ask ourselves whether an important condition for "movement detection" is met there, namely the orderly representation of sensory systems of the surface of the cerebellar cortex, which would make movement detected in the cerebellum correspond to real movement of something in the outside world.

A complicated system of maps of the body on the anterior lobe of the cerebellum of the cat has been described by Oscarsson and his group in Lund (e.g. Oscarsson 1980). The orientation of these maps, and hence their relation to the parallel fibre system varies and so does the scale of the representation. On the basis of these maps it is possible to determine to what velocities in the cat's body the intrinsic 
velocity of the cerebellum corresponds. This is, for one of the maps, a velocity of a few hundred $\mathrm{m} / \mathrm{s}$, perhaps the velocity of passive mechanical propagation of shock waves through the animal's body.

But other kinds of projection in different regions of the cerebellum have been described by Welker (1987). Here, the physiologist proceeding in the direction of parallel fibres through the cerebellar cortex does not find a continuously varying relation with some sensory map, but a surprisingly fragmented projection of various parts of the animals body.

The two observations suggest that the detector of sequential input in the cerebellum may at one time detect real movement, at another something rather of the nature of sequences of disparate events.

\section{Conclusion}

Inferences from geometry are likely to be safe inferences in the cerebellum, since many physiological details are known about synaptic interaction of neurons there. However, plasticity, i.e. changes in the neuronal interconnections in the cerebellum as a consequence of experience, is not well known yet although undoubtedly it plays a crucial role. Therefore it would be premature to propose a complete functional model of the cerebcllum.

On the basis of the observations made in the present paper I suggest the following idea. The cerebellum responds to sequences of events (in the motor, somatosensory, other sensory systems, but in other parts of the brain as well) which it picks up because they happen exactly in the correct temporal order and at the right speed of succession. Such a sequence not only activates a "beam" of parallell fibres, but in doing so furnishes, through successive activation of Purkinje cells, the sequence of signals which are needed to deal with those events. In particular, it is likely that the cerebellum thus counteracts the complicated patterns of inertial forces which are produced by any active movement of the body. It does so by exerting a series of inhibitory influences on the motor system: the output neurons of the cerebellar cortex, the Purkinje cells, are inhibitory. Relaxing the tension in a muscle that pulls in the same direction as a passive mechanical force arising during a movement is more economical than counteracting the force by an antagonist. This way of dealing with the intertial corollaries of movement is well compatible with Houk's (1987) idea of the cerebellum as an adjustable pattern generator, as well as with myographyc observations on sequential activation of muscles (e.g. Wadman et al., 1979) during the execution of simple limb movements.

\section{Acknowledgement}

Thanks are due to Hurbert Prei $\beta 1$, Sonja Grün and Dellef Heck for discussions and advice, and to Margarete Ghasroldashti and Claudia Martin-Schubert for preparing the manuscript and the pictures. 


\section{References}

Braitenberg V. (1961): "Functional interpretation of cercbellar histology." Nature 190: 539-640.

Braitenberg V. (1967): "Is the cerebellar cortex a biological clock in the millisecond range?" pp 334-346. In: Progress in Brain Rescarch, Vol. 25, The Cerebellum. Fox C.A., Snider R.S. (eds.), Elscvicr Publ. Company, Amsterdam.

Braitenberg V. (1983): "The cerebellum revisited." J. Theor. Neurobiol. 2: 237-241.

Braitenberg V., Atwood RP.(1958): "Morphological observations on the cerebellar cortex." J. Comp. Ncur. 109: 1-33.

Braitenberg V., Onesio N. (1962): The cerebellar cortex as a timing organ. Discussion of an hypothesis." pp 3-19. In: Proc. 1st Intern. Conference on Medical Cybemetics, Giannini Editore, Naples. Eccles J.C., Ito M., Szentagothai J. (1967): The cerebcllum as a neuronal machine. Springer, Berlin, Heidelberg, New York.

Houk J.C. (1987) : Model of the cerebellum as an array of adjustable pattern generators, pp 249-260. In: Cerebellum and neuronal plasticity. Glickstein M., Yeo C., Stein J. (eds.), Plenum Press, New York, London.

Oscarsson O. (1980): "Functional organization of olivary projection to the cerebellar anterior lobe", pp 279-289. In: The inferior olivary nucleus: anatomy and physiology. Courville J., de Montigny C., Lamarre Y. (eds.), Raven Press, New York.

Wadman W.J., Denier van der Gon J.J., Geuze R.H., Mol C.R. (1979): "Control of fast goal-directed arm movements." J. Human Movement Studies 5: 3-17.

Welker W. (1987): "Comparative Study of cerebellar somatosensory representations: the importance of micromapping and natural stimulation", pp 109-118. In: Cerebellum and neuronal plasticity. Glickstein M., Yeo C., Stein J. (eds.), Plenum Press, New York, London. 